University of Nebraska - Lincoln

DigitalCommons@University of Nebraska - Lincoln

Performance evaluation of spectral vegetation indices using a statistical sensitivity function

Lei Ji

USGS Center for Earth Resources Observation and Science, lji@usgs.gov

Albert J. Peters

University of Nebraska-Lincoln, apeters@calmit.unl.edu

Follow this and additional works at: https://digitalcommons.unl.edu/usgsstaffpub

Ji, Lei and Peters, Albert J., "Performance evaluation of spectral vegetation indices using a statistical sensitivity function" (2007). USGS Staff -- Published Research. 524.

https://digitalcommons.unl.edu/usgsstaffpub/524

This Article is brought to you for free and open access by the US Geological Survey at DigitalCommons@University of Nebraska - Lincoln. It has been accepted for inclusion in USGS Staff -- Published Research by an authorized administrator of DigitalCommons@University of Nebraska - Lincoln. 


\title{
Performance evaluation of spectral vegetation indices using a statistical sensitivity function
}

\author{
Lei $\mathrm{Ji}^{\mathrm{a}, *}$, Albert J. Peters ${ }^{\mathrm{b}}$ \\ ${ }^{\text {a }}$ Science Applications International Corporation (SAIC), USGS Center for Earth Resources Observation and Science (EROS), \\ Sioux Falls, SD 57198-0001, USA \\ ${ }^{\mathrm{b}}$ Center for Advanced Land Management Information Technologies (CALMIT), University of Nebraska-Lincoln, Lincoln, NE 68588-0517, USA
}

Received 31 May 2006; received in revised form 21 July 2006; accepted 21 July 2006

\begin{abstract}
A great number of spectral vegetation indices (VIs) have been developed to estimate biophysical parameters of vegetation. Traditional techniques for evaluating the performance of VIs are regression-based statistics, such as the coefficient of determination and root mean square error. These statistics, however, are not capable of quantifying the detailed relationship between VIs and biophysical parameters because the sensitivity of a VI is usually a function of the biophysical parameter instead of a constant. To better quantify this relationship, we developed a "sensitivity function" for measuring the sensitivity of a VI to biophysical parameters. The sensitivity function is defined as the first derivative of the regression function, divided by the standard error of the dependent variable prediction. The function elucidates the change in sensitivity over the range of the biophysical parameter. The Student's $t$ - or $z$-statistic can be used to test the significance of VI sensitivity. Additionally, we developed a "relative sensitivity function" that compares the sensitivities of two VIs when the biophysical parameters are unavailable.
\end{abstract}

(C) 2006 Elsevier Inc. All rights reserved.

Keywords: Vegetation index; Biophysical parameter; Sensitivity function; Regression

\section{Introduction}

\subsection{Spectral vegetation indices and biophysical parameters}

A spectral vegetation index (VI) is usually a single number derived from the spectral reflectance of two or more wavebands. Because a VI is proportional to the value of biophysical parameters such as the leaf area index (LAI), green vegetation fraction (GVF), net primary productivity (NPP), and fraction of absorbed photosynthetically active radiation (APAR), it is commonly used to indicate vegetation vigor and amount. A large number of spectral VIs have been developed and used in remote sensing. Well-known VIs include the ratio vegetation index (RVI) or simple ratio (SR) (Jordan, 1969), normalized difference vegetation index (NDVI) (Rouse et al., 1974), soil adjusted vegetation index (SAVI) (Huete, 1988), transformed

\footnotetext{
* Corresponding author. Tel.: +1 605594 6584; fax: +1 6055946529 .
}

E-mail address: 1ji@usgs.gov (L. Ji).
SAVI (TSAVI) (Baret \& Guyot, 1991), atmospherically resistant vegetation index (ARVI) (Kaufman \& Tanré, 1992), global environmental monitoring index (GEMI) (Pinty \& Verstraete, 1992), modified SAVI (MSAVI) (Qi et al., 1994), enhanced vegetation index (EVI) (Huete et al., 1994; Huete et al., 1997), optimized SAVI (OSAVI) (Rondeaux et al., 1996), green ARVI (GARI) (Gitelson et al., 1996), green NDVI (GNDVI) (Gitelson et al., 1996), etc. Additionally, other VIs proposed in recent years, such as the generalized SAVI (GESAVI) (Gilabert et al., 2002), visible atmospherically resistant index (VARI) (Gitelson et al., 2002), modified nonlinear vegetation index (MNLI) (Gong et al., 2003), linearized vegetation index (LVI) (Ünsalan \& Boyer, 2004), and wide dynamic range vegetation index (WDRVI) (Gitelson, 2004; Viña et al., 2004), have the potential for extensive application.

\subsection{Measuring the VI sensitivity to biophysical parameters}

Each spectral VI has its own merits and limitations. An ideal VI should be highly sensitive to biophysical parameters while 
being relatively insensitive to noise caused by canopy background and atmospheric effects. Previous studies have frequently used regression techniques to evaluate the effectiveness of various VIs as predictors of biophysical parameters (e.g., Gong et al., 2003; Haboudane et al., 2004; Lawrence \& Ripple, 1998; Purevdorj et al., 1998). In general, for a bivariate regression model of a biophysical parameter (independent variable $x$ ) and a VI (dependent variable $y$ ), goodness-of-fit measures such as the coefficient of determination $\left(R^{2}\right)$, mean squared error (MSE), and root mean squared error (RMSE) are useful for indicating the sensitivity of the VI to the biophysical parameter. Most investigators prefer $R^{2}$ because it represents the proportion of variability explained by the linear regression model. In addition, $R^{2}$ ranges between 0 and 1 making it a standardized measure of goodness-of-fit. Nevertheless, $R^{2}$ has a drawback in measuring goodness-of-fit because it is a function of the range and variance of samples (Achen, 1982). On a bivariate scatterplot, the range and variance of samples determine the shape of the sample cloud, which can in turn influence the $R^{2}$ value. Caution is also required when using the $R^{2}$ statistic in nonlinear regression because it can be a negative value. Therefore, instead of using $R^{2}$ in nonlinear regression, the pseudo- $R^{2}$ statistic is recommended, but pseudo- $R^{2}$ should not be interpreted as the proportion of variability explained by the nonlinear model (Schabenberger \& Pierce, 2002). MSE and RMSE are more precise indicators of sensitivity because they measure the closeness of the data and the fitted line, but sometimes MSE and RMSE are not suitable for cross-site comparison because they depend on the unit or magnitude of the measurement.

In general, $R^{2}$, MSE, and RMSE are useful for VI sensitivity analysis. These statistics, however, are just single values that summarize the overall relationship of a VI to biophysical parameters. In reality, these single values may be too simple to properly quantify the relationship because the sensitivity of a VI to biophysical parameters may change substantially with vegetation density. Fig. 1A illustrates a hypothetical example, where $x$ represents a biophysical parameter (e.g., LAI) and $y$ represents a VI (e.g., NDVI). An exponential model, $\hat{y}=0.8302$ $\left(1-\mathrm{e}^{-1.0971 x}\right)$, was found to fit the data well with a pseudo- $R^{2}$ of 0.826 and RMSE of 0.091 . But, notice that the sensitivity of $y$ to $x$ varies over the range of $x$. When $x$ is lower than 1 , a small change in $x$ corresponds to a large change in $y$, indicating a higher sensitivity of $y$ to $x$. The sensitivity is lower when $x$ is greater than 1 because a large change in $x$ produces a small change in $y$. When $x$ is larger than 3.5 , the rate of change in $y$ with respect to $x$ is close to zero, indicating that $y$ is not sensitive to $x$. In general, the sensitivity of a VI to a biophysical parameter is not a constant value, but is a function of the biophysical parameter. Thus, identification of a sensitivity function could offer more precise insight into the relationship between VIs and biophysical parameters.

Baret and Guyot (1991) developed the relative equivalent noise (REN) descriptor for evaluating the sensitivity of VI to LAI and APAR based on simulated data. The REN is defined as

$\mathrm{REN}=\frac{\sigma / x}{\mathrm{~d} \hat{y} / \mathrm{d} x}$,
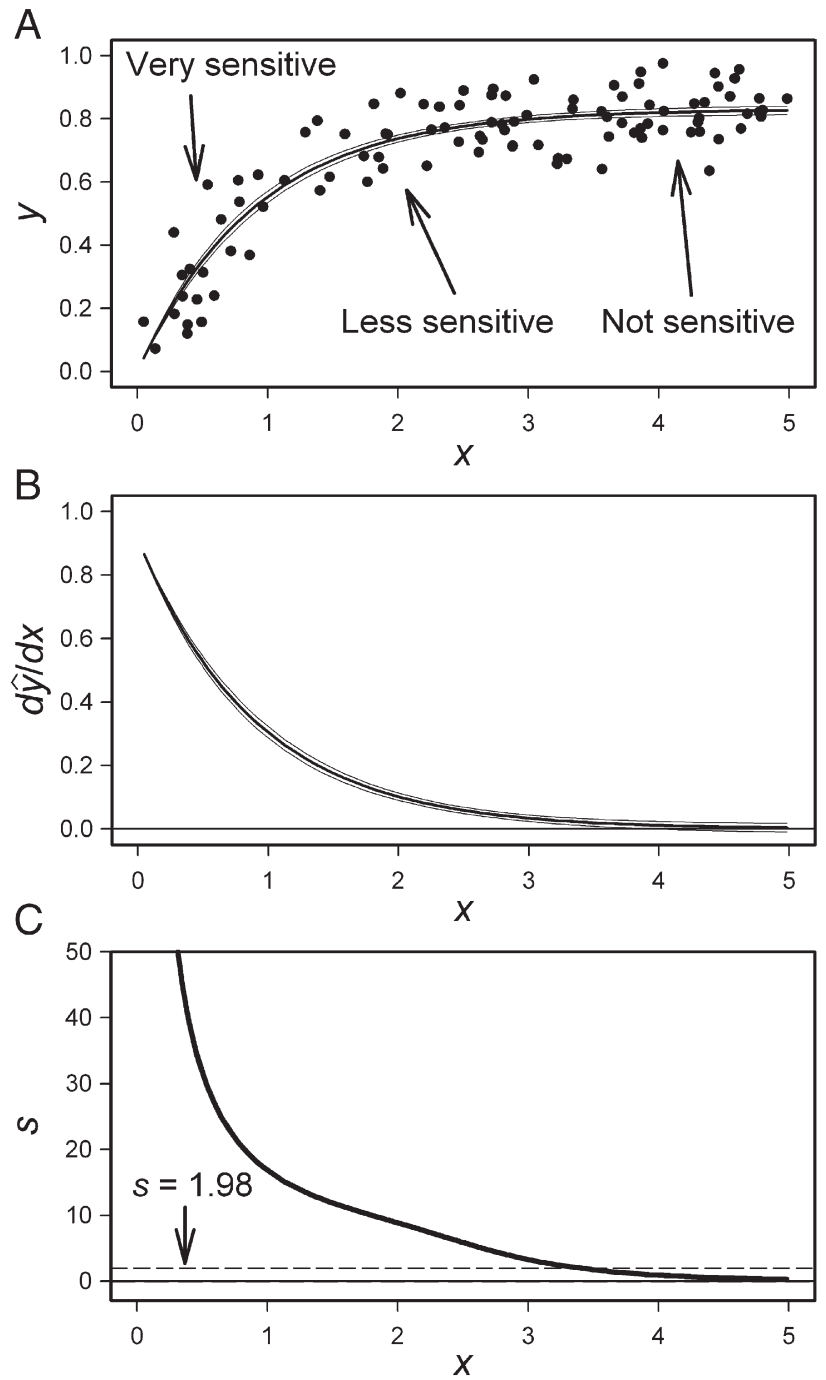

Fig. 1. Regression and sensitivity function for a hypothetical data set with 100 observations. (A) Scatterplot of $y$ versus $x$ and the exponential model fit (thick line). The thin lines are the \pm 1 standard error of $\hat{y}$. (B) The first derivative of the regression function (dŷd $/ \mathrm{d} x$ ) (thick line) and \pm 1 standard error of $\hat{y}^{\prime}$ (thin line). (C) Sensitive function $(s)$. When $s \geq 1.98, t$-score $>1.98$, and $p$-value $<0.05$, implying that the null hypothesis $H_{0}: \hat{y}^{\prime}=0$ is rejected, or the sensitivity of $y$ to $x$ is significant.

where $x$ is a biophysical parameter (LAI or APAR), $y$ is a VI (NDVI or another index), $\sigma$ is the RMSE for regression $\hat{y}=f(x)$, $\mathrm{d} \hat{\mathrm{y}} / \mathrm{d} x$ is the first derivative of the regression function, and $\sigma / x$ represents the relative error or noise. A higher REN value indicates higher noise (or lower sensitivity) for the VI. The problem with REN is that the calculation does not take the random variation of $\mathrm{d} \hat{y} / \mathrm{d} x$ into consideration. Since $\hat{y}$ values are estimated, there are estimation errors associated with $\hat{y}$ and hence the random variation of dy $/ \mathrm{d} x$. In Eq. (1), the $\sigma$ value derived from the regression does not represent the random variation of $\mathrm{d} \hat{\mathrm{y}} / \mathrm{d} x$ because $\sigma$ is a constant.

Alternatively, Huete et al. (1994) employed the vegetation equivalent noise (VEN) to represent noise in VI:

$\mathrm{VEN}=\frac{\varepsilon}{\mathrm{d} \hat{y} / \mathrm{d} x}$, 
where $x$ is a biophysical parameter, $y$ is a VI, $\varepsilon$ is the noise of $y$ on $x$ derived from the field experiment and data simulation for various soil backgrounds and atmospheric contentions. Same as REN, the VEN is expressed as the function of $x$. But, quantification of $\varepsilon$ relies on a large amount of field measurements.

Based on the regression function of two rescaled VIs, Becker and Choudhury (1988) developed the relative sensitivity $(R)$, given by

$R=\left|\frac{\mathrm{d} Y}{\mathrm{~d} X}\right|$,

where $\mathrm{d} Y / \mathrm{d} X$ is the first derivative of the regression of $Y$ against $X ; X$ and $Y$ are the rescaled VI, defined as

$X=\left(x-x_{\mathrm{s}}\right) /\left(x_{\mathrm{c}}-x_{\mathrm{s}}\right)$,

and

$Y=\left(y-y_{\mathrm{s}}\right) /\left(y_{\mathrm{c}}-y_{\mathrm{s}}\right)$,

where $x$ is the value of a VI, $x_{\mathrm{s}}$ and $x_{\mathrm{c}}$ are the values of the VI for bare soil and full canopy, respectively, and $y, y_{\mathrm{s}}$, and $y_{\mathrm{c}}$ are corresponding values of another VI. Values of $R<1,=1$, and $>1$ represent that the sensitivity of $y$ to vegetation density is respectively less than, equal to, and higher than $x$. We note that $\mathrm{d} Y / \mathrm{d} X$ is associated with random variation because $Y$ is subject to the estimation error ( $Y$ is more appropriately denoted as $\hat{Y}$ ). Thus, a measure of sensitivity using $R$ may not be accurate because the random variation of $\mathrm{d} Y / \mathrm{d} X$ is not accounted for in the formulation. Gitelson (2004) proposed another relative sensitivity $\left(S_{\mathrm{r}}\right)$, which is defined as

$S_{\mathrm{r}}=\left(\frac{\mathrm{d} \hat{y}}{\mathrm{~d} x}\right)\left(\frac{\Delta x}{\Delta y}\right)$,

where $x$ and $y$ denote two VIs, $\mathrm{dy} / \mathrm{d} x$ is the first derivative of the regression function $\hat{y}=f(x)$, and $\Delta x$ and $\Delta y$ are the ranges (the maximum value minus the minimum value) of $x$ and $y$, respectively. An $S_{\mathrm{r}}$ value $<1,=1$, or $>1$ indicates the relative sensitivity of $y$ is less than, equal to, or more than $x$, respectively. Like Becker and Choudhury's $R, S_{\mathrm{r}}$ does not account for the estimation errors of $\mathrm{dy} / \mathrm{d} x$, which may bias the evaluation of sensitivity.

\subsection{Research objectives}

As discussed above, previously used measures such as $R^{2}$ and RMSE are useful for sensitivity analysis, but they provide only a generalized summary of the relationships between VIs and biophysical parameters. In general, the sensitivity of a VI to a biophysical parameter should not be just a constant, but rather a function of the biophysical parameter. REN and VEN do provide measures of sensitivity/noise that appear as functions of the biophysical parameters, but random errors of the derivative are not incorporated into the calculation of sensitivity/noise. The objective of this research was to develop a new "sensitivity function" that can (a) indicate the change in sensitivity over the range of a biophysical parameter, (b) take estimation error of the regression function into account regarding sensitivity over a range of conditions, and (c) offer a statistical test for sensitivity. In addition, we created a "relative sensitivity function" for directly comparing two VIs based on the regression of the two indices.

\section{Development of sensitivity function}

\subsection{Sensitivity function}

Suppose we have a set of measurements on both a biophysical parameter (e.g., LAI) and a spectral VI (e.g., NDVI). Our goal is to depict the sensitivity of NDVI to LAI over the entire range of LAI. We can build a bivariate regression model using LAI as the independent variable $(x)$ and NDVI as the dependent variable $(y)$. The fitted regression function is given by

$\hat{y}=f(x)$.

The regression function can be linear, curvilinear, or nonlinear. Since $\hat{y}$ values are estimated, they have an associated estimation error, represented by the standard error of $\hat{y}$, denoted as $\sigma_{\hat{y}}$. In linear regression and curvilinear models, $\sigma_{\hat{y}}$ is given by

$\sigma_{\hat{y}_{i}}=\sqrt{\sigma^{2} \mathbf{X}_{i}^{\prime}\left(\mathbf{X}^{\prime} \mathbf{X}\right)^{-1} \mathbf{X}_{i}}$,

where $\sigma^{2}$ is the MSE, $\mathbf{X}$ is the matrix of independent variables, and $\mathbf{X}_{i}$ is the $i$ th row of $\mathbf{X}$. In nonlinear regression, the standard error of $\hat{y}_{i}$ is approximated using the asymptotic standard error, given by

$\sigma_{\hat{y}_{i}}=\sqrt{\sigma^{2} \mathbf{F}_{i}^{\prime}\left(\mathbf{F}^{\prime} \mathbf{F}\right)^{-1} \mathbf{F}_{i}}$,

where $\mathbf{F}$ is the matrix of derivatives for approximating least squares estimations and $\mathbf{F}_{i}$ denotes the $i$ th row of $\mathbf{F}$ matrix (Schabenberger \& Pierce, 2002). More detailed definitions and explanations of $\mathbf{X}$ and $\mathbf{F}$ matrices can be found in regression textbooks. Although calculation of the standard error is complicated, especially for nonlinear models, we can rely on statistical analysis packages to accomplish the computation.

The first derivative $\left(\hat{y}^{\prime}\right)$ of the regression function (Eq. (7)) is expressed as

$\hat{y}^{\prime}=f^{\prime}(x)=\frac{\mathrm{d}(\hat{y})}{\mathrm{d}(x)}$.

Here, $\hat{y}^{\prime}$ reflects the rate of the change in $\hat{y}$ versus the change in $x$. That is, $\hat{y}^{\prime}$ measures how many units of change in $\hat{y}$ for every one unit of change in $x$ at a given $x$ value. Note that $\hat{y}^{\prime}$ measures the sensitivity of $x$ to $\hat{y}$, rather than to $y$. Therefore, it is inadequate to use $\hat{y}^{\prime}$ as an indicator of sensitivity, unless the variability of $\hat{y}^{\prime}$, or the standard error of $\hat{y}^{\prime}$ (denoted as $\sigma_{\hat{y}^{\prime}}$ ) is incorporated into the calculation. Because in regression (Eq. (7)) the independent variable $x$ is assumed to be fixed, the standard error of $\hat{y}^{\prime}$ equals the standard error of $\hat{y}$, i.e., $\sigma_{\hat{y}^{\prime}}=\sigma_{\hat{y}}$. Now we can define the sensitivity function $(s)$ as

$s=\frac{\hat{y}^{\prime}}{\sigma_{\hat{y}}}=\frac{\mathrm{d} \hat{y} / \mathrm{d} x}{\sigma_{\hat{y}}}$. 

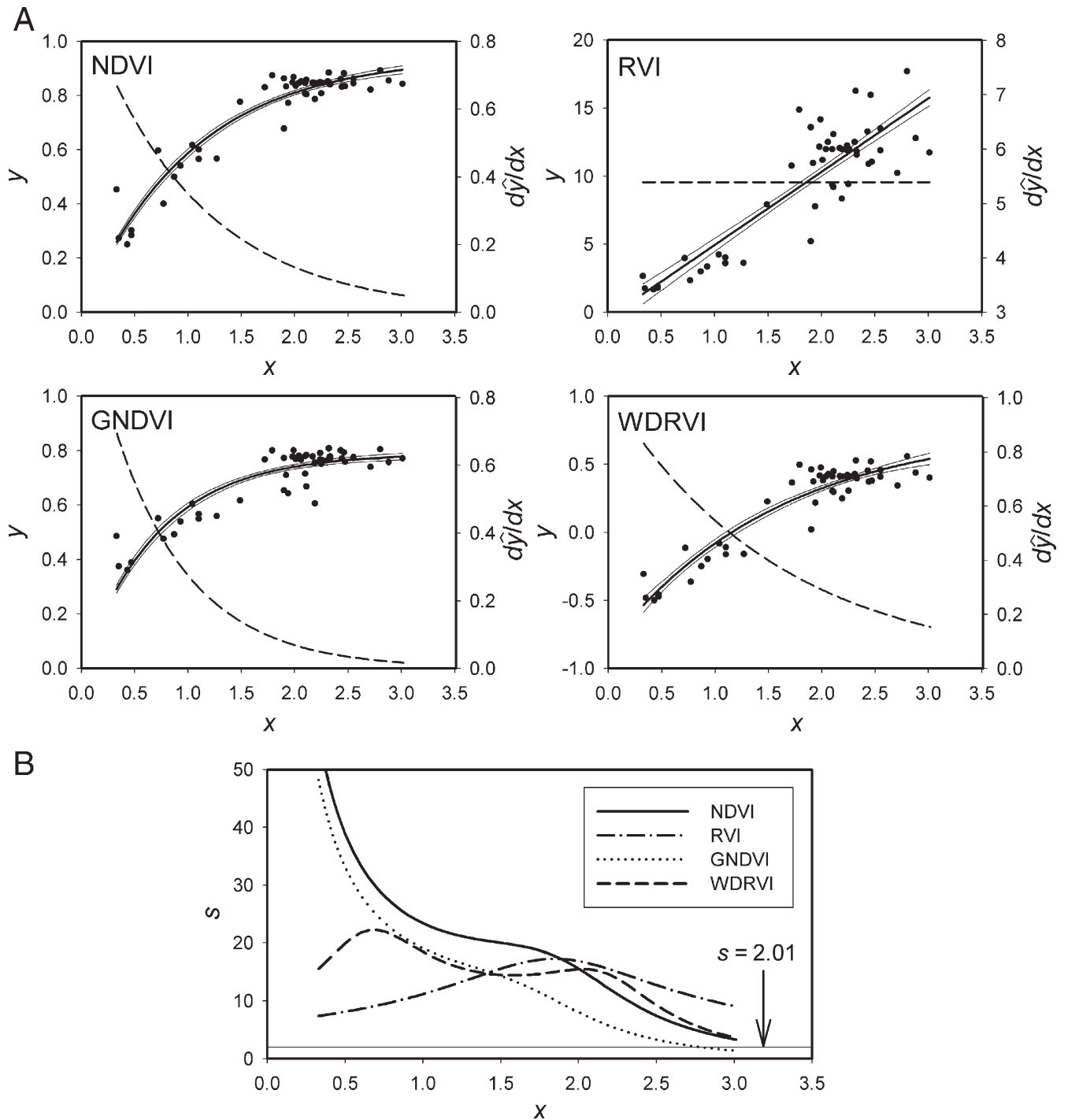

Fig. 2. Sensitivity analysis for vegetation indices and green LAI measured in a corn field. (A) Scatterplots (solid circles), regression function (thick solid lines), standard errors of the regression function (thin solid lines), and first derivatives of the regression functions (dashed lines) for the vegetation indices (dependent variable $y$ ) and LAI (independent variable $x$ ). (B) Sensitivity functions $(s)$ for the four vegetation indices. The sensitivity of a vegetation index to LAI (denoted as $x$ ) is significant when $s>2.01$.

Large positive $s$-values represent a high sensitivity of $x$ to $y$, and negative $s$-values indicate an inverse sensitivity. In this equation, the unit of $s$ is $x^{-1}$, or the reciprocal of $x$. Thus, the $s$-value is irrelevant to the magnitude of the VI, but dependent on the unit or magnitude of the biophysical parameter. As a result, use of the sensitivity function is appropriate for comparing sensitivity among VIs having different magnitudes or dynamic ranges. Additionally, caution is required if sensitivities of VIs are compared over the entire range of the values for the biophysical parameter. Due to the impact of canopy background (e.g., soil color, brightness, wetness, etc.) on spectral signals, the sensitivity may show variations caused by the changes in the soil background. In this case, sensitivities of VIs need to be compared and evaluated under the same soil background.

The significance of $\hat{y}^{\prime}$ can be tested with the Student's $t$ - or $z$-statistic. For the $i$ th observation, the null hypothesis is $H_{0}: \hat{\mathrm{y}}_{i}^{\prime}$
$=0$ and the alternative hypothesis is $H_{1}: \hat{y}_{i}^{\prime} \neq 0$. The two-tail $t$ test for $\hat{y}_{i}^{\prime}$ is given by

$t=\frac{\hat{y}_{i}^{\prime}}{\sigma_{\hat{y}_{i}}}$

The result of the $t$-test is indicated by the corresponding $p$-value, which either rejects $(p$-value $<\alpha)$ or fails to reject the $H_{0}$. Note that this formula is the same as the sensitivity function (Eq. (11)). Consequently, the $s$-value itself indicates the $t$-score. Besides, we can create the confidence intervals for $\hat{y}_{i}^{\prime}$, which are given by

$\hat{y}_{i}^{\prime} \pm t_{\alpha / 2, v} \sigma_{\hat{y}}$

where $t_{\alpha / 2, v}$ is the $t$-score for degree of freedom $v$ at $\alpha$ level. 
In the example presented in Fig. 1A, we obtained the first derivative of the regression function $\left(\hat{\mathrm{y}}^{\prime}=0.8302 \mathrm{e}^{-1.0971 x}\right)$ and calculated the asymptotic standard errors for $\hat{y}_{i}$ (Fig. 1B). The sensitivity function $(s)$ was computed and plotted against $x$ (Fig. 1C). The sensitivity curve shows that the sensitivity decreases with $x$ at an exponentially decaying rate. When $x$ is greater than 3.4, the $s$-value is less than 1.98 , the critical value in the two-tailed $t$-test $(\alpha=0.05, v=98)$ for accepting $H_{0}$. We concluded that, even though the goodness-of-fit of the regression is generally high as indicted by the psuedo- $R^{2}(0.826)$ and RMSE (0.091), sensitivity is not significant in the domain of $x>3.4$.

\subsection{Relative sensitivity function}

In a case where we have two spectral VIs ( $x$ and $y$ ), but where we lack in situ measurements of biophysical parameters, we can calculate the relative sensitivity for the two VIs. First, we choose $y$ as the dependent variable and $x$ as the independent variable to create the $y$ on $x$ regression $[\hat{\mathrm{y}}=f(x)]$. The first derivative $\left(\hat{\mathrm{y}}^{\prime}\right)$ of the regression function and the standard error of $\hat{y}$ (denoted as $\sigma_{\hat{y}}$ ) are obtained by following the procedure described earlier. The sensitivity of $y$ relative to $x$ can be expressed by the relative sensitivity function $\left(s_{y \mid x}\right)$ :

$s_{y \mid x}=\frac{\hat{y}^{\prime}}{\sigma_{\hat{y}}}=\frac{\mathrm{d} \hat{y} / \mathrm{d} x}{\sigma_{\hat{y}}}$.

Then we invert $x$ and $y$ to create the $x$ on $y$ regression $[\hat{\mathrm{x}}=g(y)]$. Likewise, we calculate the first derivative $\left(\hat{x}^{\prime}\right)$ and the standard error of $\hat{\mathrm{x}}$ (denoted as $\sigma_{\hat{\mathrm{x}}}$ ). Thus, the sensitivity of $x$ relative to $y$ is represented by the relative sensitivity function $\left(s_{x \mid y}\right)$, given by

$s_{x \mid y}=\frac{\hat{x}^{\prime}}{\sigma_{\hat{x}}}=\frac{\mathrm{d} \hat{x} / \mathrm{d} y}{\sigma_{\hat{x}}}$.

Plots of $s_{y \mid x}$ and $s_{x \mid y}$ versus $x$ (or $y$ ) can demonstrate the relative sensitivity of the two VIs. We note that two relative sensitivity functions are calculated based on two separate regressions. Why are two regressions necessary? For an ordinary regression model, one assumption is that the independent variable is fixed or controlled and the dependent variable is subject to error. The regression estimation minimizes the sum of squared errors in the dependent variable. If only one regression is used for evaluating the sensitivity of two VIs, the measurement error of the independent variable will be unfairly ignored. A misleading conclusion can be drawn if we assume that one VI is error-free while the other is not. Thereafter, to treat the two VIs equally and symmetrically, we should use two separate regressions to analyze sensitivity of the VIs.

\section{Use of the sensitivity function}

To demonstrate the usefulness of the sensitivity function for evaluating the performance of a VI, we now present two case studies.

\subsection{Sensitivity of VIs to green LAI for a corn canopy}

In this example, the sensitivities of four VIs to green LAI were analyzed based on close-range hyperspectral data collected in an experimental corn field during the early to middle growing season (14 June to 16 August) in 1999. The field was located at the University of Nebraska Agriculture Research and Development Center, about $60 \mathrm{~km}$ northeast of Lincoln, Nebraska. The hyperspectral data were measured over 21 corn plots with a Spectron Engineering SE-590 spectroradiometer that recorded spectral data in 252 bands with a nominal range between 356 and $1126 \mathrm{~nm}$. The sensor head, located about $6 \mathrm{~m}$ above ground, was mounted on the boom of a data collection vehicle. A white Spectralon reflectance panel was used to calibrate the spectroradiometer and convert canopy reflectance to percent reflectance. All spectral data were collected near solar noon (11 a.m. to 3 p.m.) to minimize the effect of the solar zenith angle on canopy reflectance. The NDVI, GNDVI, RVI, and WDRVI $(\alpha=0.2)$ were calculated based on percent reflectance in simulated green $(545-565 \mathrm{~nm})$, red $(620-670 \mathrm{~nm})$, and near infrared (841-876 nm) wavelengths. Green LAI of the corn canopy was measured using a LI-COR LI-2000 Area Meter on the same day as the spectral measurements. The spectral and LAI data were measured at 3 and 15 random points, respectively, in each plot, and the averaged spectral and LAI values for each plot were used in the statistical analyses.

Sensitivity analysis on VIs and LAI was conducted for 50 observations (plot by date), as shown in Fig. 2. The parameter estimate and goodness-of-fit for the regression functions of VI $(y)$ on LAI $(x)$ are indicated below:

\begin{tabular}{llll}
\hline NDVI & $\hat{y}=0.9457\left(1-\mathrm{e}^{-0.9714 x}\right)$ & $\sigma=0.0559$ & Psuedo- $R^{2}=0.910$ \\
RVI & $\hat{y}=-0.4461+5.3841 x$ & $\sigma=2.2081$ & $R^{2}=0.761$ \\
GNDVI & $\hat{y}=0.7898\left(1-\mathrm{e}^{-1.3904 x}\right)$ & $\sigma=0.0559$ & Psuedo- $R^{2}=0.826$ \\
WDRVI & $\hat{y}=-0.8395+1.6231\left(1-\mathrm{e}^{-0.6309 x}\right)$ & $\sigma=0.1091$ & Psuedo- $R^{2}=0.888$ \\
\hline
\end{tabular}

The $R^{2}$ or psuedo- $R^{2}$ values show that the regression fit for NDVI and WDRVI is better than that for GNDVI and RVI. In this case, the RMSE values are almost useless in the model comparison because the magnitudes of the VIs are different. Based on the first derivative of regression $\left(\hat{\mathrm{y}}^{\prime}\right)$ and the standard errors of the regression function $\left(\sigma_{\hat{y}}\right)$, as shown in Fig. 2A, we obtained the sensitivity functions $(s)$ for the four VIs (Fig. 2B). Among the four VIs, NDVI has the highest sensitivity when LAI is less than 1.8, and RVI has the highest sensitivity when LAI is greater than 1.8. Although GNDVI is sensitive to LAI when LAI is less than 1.4, the index performs poorly when LAI is greater than 1.4. The sensitivity of WDRVI is relatively consistent over the entire range of LAI, but it is inferior to NDVI (when $\mathrm{LAI}<2.0$ ) and RVI (when LAI $>2.0$ ). The $t$-statistic shows that the $t$-scores (same as $s$-values) are all greater than 2.01 for the four VIs, except GNDVI which has the $s$-value less than 2.01 when LAI $>2.8$. Here, $t$-score of 2.01 is the critical value in the two-tail $t$-test $(\alpha=0.05, v=48)$ for ejecting the $H_{0}: \hat{y}^{\prime}=0$. Thus, we conclude that among the four VIs, all are significantly sensitive to LAI, except GNDVI, which is not significant when LAI is high. 


\subsection{Relative sensitivity of satellite-derived NDVI and EVI}

Our second example is the sensitivity analysis for the NDVI and EVI data acquired with the Moderate Resolution Imaging Spectroradiometer (MODIS) instrument. The two data sets are the 1-km resolution 16-day composites (MOD13A2, version 4) derived from MODIS on the Terra platform (http://pdaac.usgs. gov/modis/mod13a2v4.asp). The Quality Assurance data were used to identify cloud, shadow, and snow/ice pixels, which were excluded in the data analysis. Land cover types were identified using the MODIS/Terra Global $1 \mathrm{~km}$ Land Cover Data (MOD12Q1, Type 1). We selected one deciduous broad-leaf forest site centered at $60^{\circ} 34^{\prime} \mathrm{N}$ to $103^{\circ} 09^{\prime} \mathrm{W}$ in the North Central Appalachians Ecoregion, based on the map of the Level III
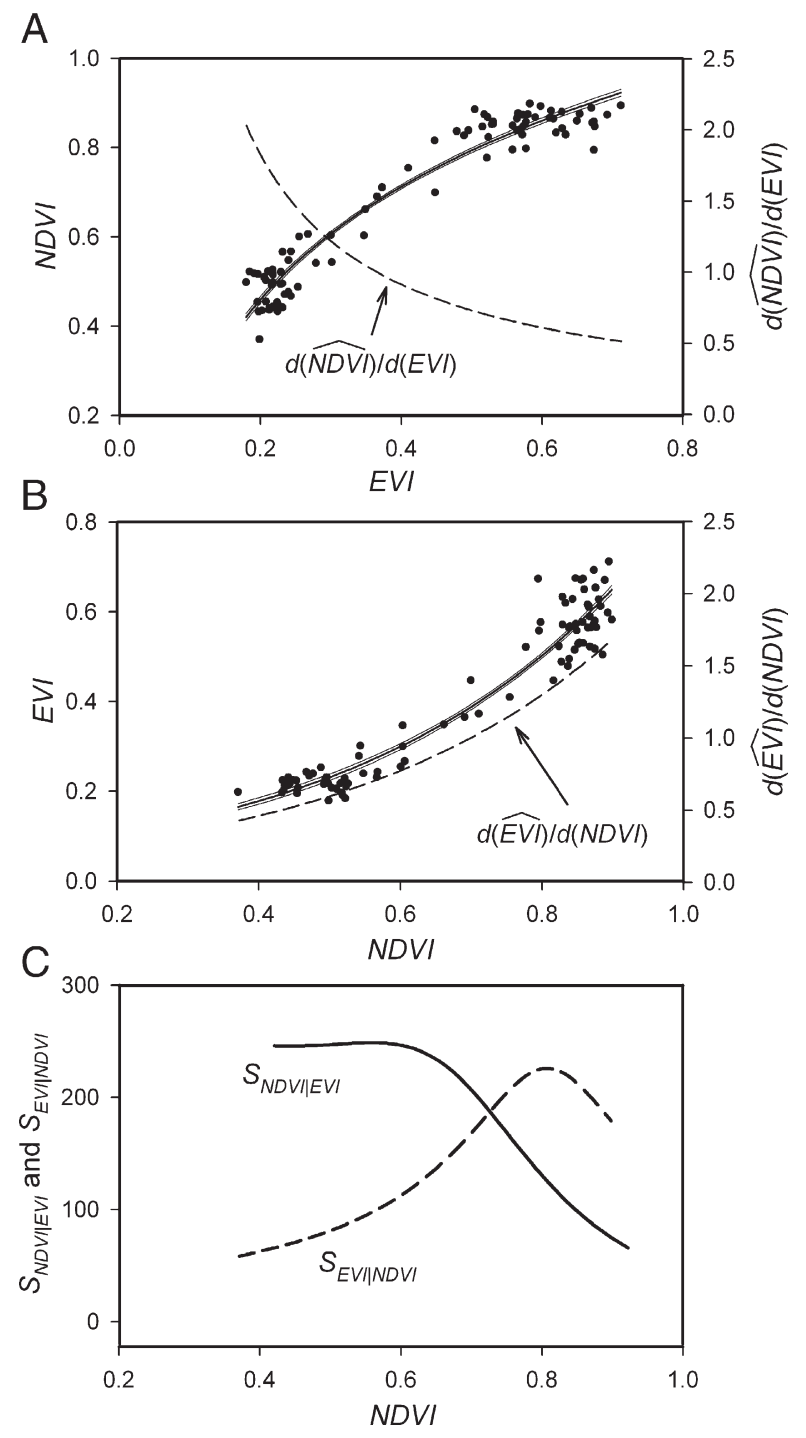

Fig. 3. Sensitivity analysis for MODIS NDVI and EVI at a deciduous forest site in the North Central Appalachians Ecoregion. (A) NDVI on EVI regression function (thick solid line), one standard error (thin solid line), and first derivative (dashed line). (B) EVI on NDVI regression function (thick solid line), one standard error (thin solid line), and first derivative (dashed line). (C) Relative sensitivity of NDVI to EVI $\left(s_{\mathrm{NDVI} \mid \mathrm{EVI}}\right)$ and relative sensitivity of EVI to NDVI $\left(s_{\mathrm{EVI} \mid \mathrm{NDVI}}\right)$
Ecoregions of the Continental United States (Omernik, 1987). The sampling site was a $12 \times 12 \mathrm{~km}^{2}$ and the median values of NDVI and EVI in the square were used for the sensitivity analysis. We analyzed 94 composite intervals between 18 February 2000 and 31 December 2004. Some composite intervals were not analyzed due to cloud/shadow contamination or snow/ice conditions.

The relationship between NDVI $(y)$ and EVI $(x)$ was explored by using logarithmic and exponential regression models (Fig. 3A and $\mathrm{B})$ :

\begin{tabular}{llll}
\hline NDVI on EVI & $\hat{y}=1.0466+0.3649 \ln (x)$ & $\sigma=0.0440$ & Psuedo- $R^{2}=0.940$ \\
EVI on NDVI & $\hat{x}=0.0635 \mathrm{e}^{2.5874 y}$ & $\sigma=0.0493$ & Psuedo- $R^{2}=0.926$ \\
\hline
\end{tabular}

Based on the first derivatives and standard errors of $\hat{y}$ and $\hat{\mathrm{x}}$ (Fig. 3A and B), we obtained the relative sensitivity functions $s_{y \mid x}$ and $s_{x \mid y}$ (Fig. 3C). The relative sensitivity functions indicate that the relative sensitivity of NDVI to EVI is high when NDVI is less than 0.72 , and the relative sensitivity of EVI to NDVI is high when NDVI is greater than 0.72 . This suggests that NDVI is more sensitive to low-density canopy and EVI is more sensitive to high-density canopy in this region.

\section{Conclusions}

To meet the need for assessing the performance of VIs, we developed a sensitivity function based on the regression analysis for VIs and biophysical parameters. The sensitivity function is calculated from the ratio of the first derivative to the standard error of the regression function. The sensitivity function has several advantages: (1) it tracks the changes in sensitivity of a VI through the range of biophysical variables, rather than providing a single goodness-of-fit value; (2) it is irrelevant of the unit or magnitude of VIs, thus making it possible to compare various VIs; and (3) it tests the significance of the sensitivity with the $t$ - or $z$-statistic. The relative sensitivity function, obtained by using the regressions of the two VIs, is designed for direct comparison of two VIs. Broadly, the sensitivity analysis technique is not only suitable for the investigation of VIs, but can also be extended to other climatic, ecological, and environmental variables.

In this paper, two case studies are provided to demonstrate uses for the sensitivity function. Our goal in the paper was to introduce a technique for sensitivity analysis of VIs, rather than provide a comprehensive comparison of VIs. A thorough evaluation of the performance of VIs will require an extensive and profound investigation of remotely sensed data and ground measurements.

\section{Acknowledgement}

This work was initially supported by the NASA Affiliated Research Center Program under Cooperative Agreement NCC1317. The field data were collected by the field research team-Joel Connot, Dave Derry, Juan Ramirez, John Vidlak, and Andres Viña-at the University of Nebraska-Lincoln, Center for Advanced Land Management Information Technologies. We would like to thank Eugene Fosnight, Donald Rundquist, and Bruce 
Wylie for reviewing the manuscript and offering useful comments and suggestions.

\section{References}

Achen, C. H. (1982). Interpreting and using regression. Sage university paper series on quantitative application in the social sciences $07-001$. Beverly Hills: Sage Publications, 85 pp.

Baret, F., \& Guyot, G. (1991). Potentials and limits of vegetation indices for LAI and APAR assessment. Remote Sensing of Environment, 35, 161-173.

Becker, F., \& Choudhury, B. J. (1988). Relative sensitivity of normalized difference vegetation index (NDVI) and microwave polarization difference index (MPDI) for vegetation and desertification monitoring. Remote Sensing of Environment, 24, 297-311.

Gilabert, M. A., González-Piqueras, J., García-Haro, F. J., \& Meliá, J. (2002). A generalized soil-adjusted vegetation index. Remote Sensing of Environment, 82, 303-310.

Gitelson, A. A. (2004). Wide dynamic range vegetation index for remote quantification of biophysical characteristics of vegetation. Journal of Plant Physiology, 161, 165-173.

Gitelson, A. A., Kaufman, Y. J., \& Merzlyak, M. N. (1996). Use of a green channel in remote sensing of global vegetation from EOS-MODIS. Remote Sensing of Environment, 58, 289-298.

Gitelson, A. A., Kaufman, Y. J., Stark, R., \& Rundquist, D. (2002). Novel algorithms for remote estimation of vegetation fraction. Remote Sensing of Environment, 80, 76-87.

Gong, P., Pu, R., Biging, G. S., \& Larrieu, M. R. (2003). Estimation of forest leaf area index using vegetation indices derived from hyperion hyperspectral data. IEEE Transactions on Geoscience and Remote Sensing, 40, 1355-1362.

Haboudane, D., Miller, J. R., Pattey, E., Zarco-Tejada, P. J., \& Strachan, I. B. (2004). Hyperspectral vegetation indices and novel algorithms for predicting green LAI of crop canopies: Modeling and validation in the context of precision agriculture. Remote Sensing of Environment, 90, 337-352.

Huete, A. R. (1988). A soil-adjusted vegetation index (SAVI). Remote Sensing of Environment, 25, 295-309.
Huete, A., Justice, C., \& Liu, H. (1994). Development of vegetation and soil indices for MODIS-EOS. Remote Sensing of Environment, 49, 224-234.

Huete, A. R., Liu, H. Q., Batchily, K., \& van Leeuwen, W. (1997). A comparison of vegetation indices over a global set of TM images for EOS-MODIS. Remote Sensing of Environment, 59, 440-451.

Jordan, C. F. (1969). Derivation of leaf-area index from quality of light on the forest floor. Ecology, 50, 663-666.

Kaufman, Y. J., \& Tanré, D. (1992). Atmospherically resistant vegetation index (ARVI) for EOS-MODIS. IEEE Transactions on Geoscience and Remote Sensing, 30, 261-270.

Lawrence, R. L., \& Ripple, W. J. (1998). Comparisons among vegetation indices and bandwise regression in a highly disturbed, heterogeneous landscape: Mount St. Helens, Washington. Remote Sensing of Environment, 64, 91-102.

Omernik, J. M. (1987). Ecoregions of the conterminous United States. Annals of the Association of American Geographers, 77, 118-125.

Pinty, B., \& Verstraete, M. M. (1992). GEMI: A non-linear index to monitor global vegetation from satellites. Vegetatio, 101, 15-20.

Purevdorj, Ts., Tateishi, R., Ishiyama, T., \& Honda, Y. (1998). Relationships between percent vegetation cover and vegetation indices. International Journal of Remote Sensing, 19, 3519-3535.

Qi, J., Chehbouni, A., Huete, A. R., Kerr, Y. H., \& Sorooshian, S. (1994). A modified soil adjusted vegetation index. Remote Sensing of Environment, 48, 119-126.

Rondeaux, G., Steven, M., \& Baret, F. (1996). Optimization of soil-adjusted vegetation indices. Remote Sensing of Environment, 55, 95-107.

Rouse, Jr., J.W., Haas, R. H., Shell, J. A., \& Deering, D. W. (1974). Monitoring vegetation systems in the Great Plains with ERTS. Third earth resources technology satellite-1 symposium, Vol. 1, 10-14 December, 1973 (pp. 309-317). Washington, D. C.: NASA Scientific and Technical Information Office.

Schabenberger, O., \& Pierce, F. J. (2002). Contemporary statistical models for the plant and soil sciences. Boca Raton, Florida: CRC Press, 738 pp.

Ünsalan, C., \& Boyer, K. L. (2004). Linearized vegetation indices based on a formal statistical framework. IEEE Transactions on Geoscience and Remote Sensing, 42, 1575-1585.

Viña, A., Henebry, G. M., \& Gitelson, A. A. (2004). Satellite monitoring of vegetation dynamics: Sensitivity enhancement by the wide dynamic range vegetation index. Geophysical Research Letters, 31, L04503. 